\title{
Efectos del tape dinámico en la función dinámica y estática del tobillo en mujeres con características de pie pronador, Pereira 2019
}

Investigación en Curso

\author{
Maricela Bustamante Betancourt'; Nathalia Alejandra Peláez Duque'; \\ Alejandro Gómez Rodas ${ }^{2}$
}

RESUMEN Introducción: diversos cambios biomecánicos ocurren al caminar y estar de pie. A lo largo de la zancada normal, el pie se mueve desde una posición pronada a una posición supinada, lo que permite amortiguar las fuerzas de reacción y adaptación al terreno. Diversas estrategias se han planteado y utilizado para brindar una mejoría de la pronación excesiva, la cual se ha relacionado con numerosos cambios funcionales de la extremidad inferior lo que resulta en lesiones por sobreuso. Se han desarrollado herramientas para evaluar el grado de pronación del pie incluida la prueba de caída navicular, los ángulos de rotación tibial y el índice de postura del pie. El mecanismo de acción del tapping se ha especificado en estudios anteriores; sin embargo recientemente, ha hecho su aparición un nuevo tipo de tape con capacidades de amortiguación elástica denominado tape dinámico. Por tal, motivo se hace necesario estudiar el efecto que el tape dinámico puede tener en la función estática y dinámica en mujeres con pie pronado.

Métodos: investigación de tipo explicativa de carácter cuasi experimental. Se vincularán 30 estudiantes universitarias entre los 18 y 26 años de edad con características de pie pronado asignando 15 estudiantes a un grupo al que se le aplicará tape dinámico con efecto antipronador y 15 estudiantes a los que se les aplicará tape dinámico sin tensión. Se analizarán pruebas funcionales del miembro inferior antes, inmediatamente después y a los tres días después de aplicado el tape. Se efectuará un análisis de diferencia de medias intra e intergrupos previa comprobación de la normalidad de los datos para determinar los efectos del tape dinámico en la funcionalidad dinámica y en la estática del tobillo.

Resultados esperados: el desempeño en la función dinámica y la estática del tobillo en mujeres universitarias con características de pie pronado mejora con el uso del tape dinámico.

\section{PALABRAS Cinta Atlética, \\ CLAVE $\quad$ Rendimiento Funcional, Pie Pronado}

1 Estudiantes de quinto y sexto semestre de Fisioterapia. Fundación Universitaria del Área Andina. Seccional Pereira. Integrante del semillero SEMFIS.

2 Docente Fundación Universitaria del Área Andina y Universidad Tecnológica de Pereira, Programa de Fisioterapia y Programa Ciencias del Deporte y la Recreación. 


\section{Effects of dynamic tape on the dynamic and static function of the ankle in women with pronated foot}

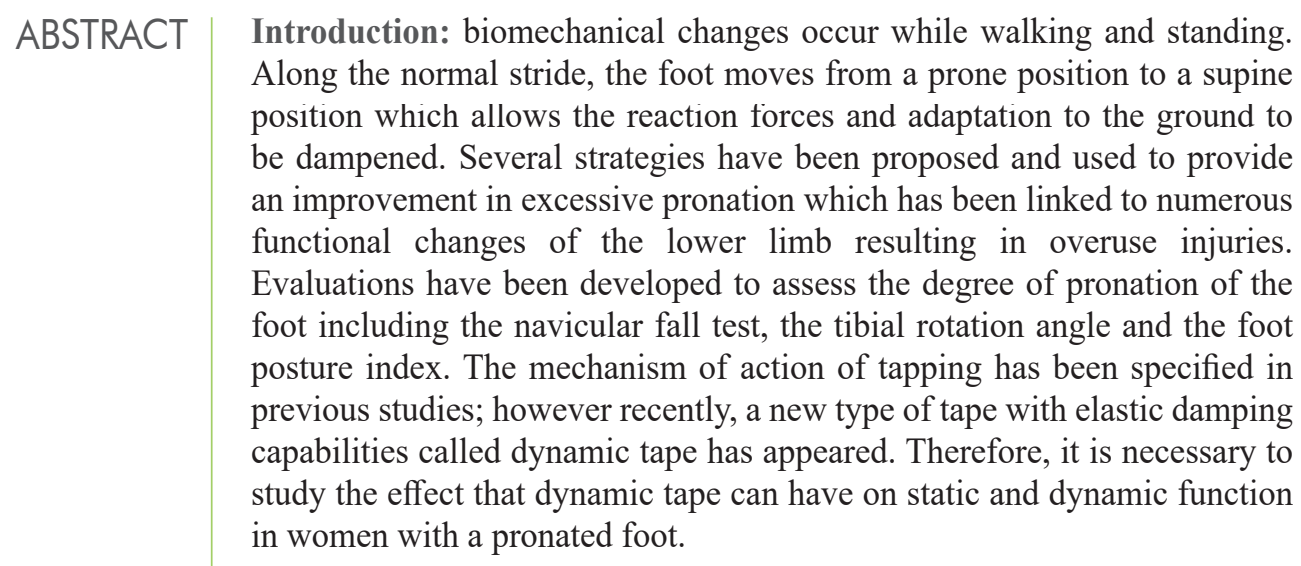

Methods: quasi-experimental explanatory research. Thirty university students between 18 and 26 years of age will be linked with pronated foot characteristics, assigning 15 students to a group that will be applied dynamic tape with antipronator effect and 15 students to whom dynamic tape will be applied without tension. Functional tests of the lower limb will be analyzed before, immediately after and three days after the tape is applied. A difference analysis of intra and intergroup means will be carried out after checking the normality of the data to determine the effects of the dynamic tape on the functionality and static of the ankle.

Expected results: the dynamic functional performance and static of the foot in university women with pronated foot characteristics improves with use of dynamic tape.

Athletic Tape,

Functional Performance,

Pronated Foot 


\section{INTRODUCCIÓN}

El pie posee un complejo articular formado por diferentes estructuras musculares, tendinosas, óseas y ligamentosas, que facilitan y dan soporte en diversos ejes de movimiento, dando dirección y permitiendo la ejecución de diferentes actividades en la vida diaria (1). Esta gran libertad de movimiento requiere diversas adaptaciones biomecánicas para posibilitar la marcha, la carrera y la posición bípeda. A lo largo de la zancada normal, el pie se mueve desde una posición pronada a una posición supinada, lo que permite amortiguar las fuerzas de reacción y adaptación al terreno (2).

La pronación hace parte del movimiento normal del pie, esta se produce típicamente entre las fases de golpe de talón y de postura media del ciclo de la marcha. Sin embargo, la pronación excesiva del pie se ha definido como una pérdida del arco longitudinal medial, mientras que las articulaciones subtalar y mediotarsiana muestran un movimiento más amplio del rango normal (3).

Durante las actividades de esfuerzo, el pie se encuentra sometido a cargas que son transmitidas al resto del cuerpo, sin embargo, la pronación es la combinación de diferentes movimientos; la abducción, eversión y flexión plantar. Pero cuando estas estructuras se alteran presentan numerosos cambios funcionales, dolor, irregularidad y desalineación del cuerpo o del miembro inferior. Esta desalineación anormal puede estirar y debilitar los músculos intrínsecos del pie, al alargarlos más allá de su posición fisiológica de reposo neutral, cambiando la relación entre la longitud y la tensión de los músculos, lo que puede impedir que los músculos generen una fuerza adecuada u óptima (4).

La pronación excesiva causa una serie de alteraciones en la biomecánica del miembro inferior, siendo esta estructura capaz de modificar la posición cuando se presenta algún desorden a nivel muscular y articular. Por consiguiente, la pronación excesiva del pie se ha relacionado con numerosos cambios funcionales en la extremidad inferior, lo que resulta en lesiones por uso excesivo asociándose con el síndrome de estrés medial de la tibia, la fascitis plantar, el hallux rigidus y el síndrome de dolor patelofemoral (5), este tipo de pie puede presentar un aumento del área de contacto medial con mayores fuerzas y presiones excesivas (4).

El mecanismo de lesión se produce a través de la propagación de mecanismos funcionales anormales proximales. La pronación de la cadena cerrada que se produce en la articulación subtalar implica la eversión del calcáneo, la aducción y la flexión plantar (5).

Se han desarrollado herramientas para evaluar el grado de pronación del pie, la estática de la postura del pie, y la evaluación dinámica de la función del pie, incluida la prueba de caída navicular, los ángulos de rotación tibial y el índice de postura del pie (FPI) (6). 
Las intervenciones utilizadas para intentar corregir la pronación excesiva incluyen tratamientos quirúrgicos, la prescripción de ortesis y el tapping, esta última ha sido de gran utilidad debido que tiene un espesor comparable a la epidermis de la piel y puede estirarse entre el 30\% y el 40\% de su longitud en reposo (7).

El kinesio taping o tapping son materiales que estimulan los mecanorreceptores localizados en la piel; estos receptores son sensoriales y reaccionan para llevar información al sistema nervioso, brindan un mejor aporte sanguíneo y contribuyen a recuperar dolores, lesiones o afecciones musculoesqueléticas, provocando un efecto de mejoría y alivio a nivel propioceptivo (8).

El método del Kinesio taping fue diseñado para promover el rango completo de movimiento. Esta cinta es porosa y permite la evaporación del sudor y el agua. El adhesivo se activa por calor aumentando su efecto después de haber sido aplicado (9).

El mecanismo de acción del tapping se ha especificado en estudios anteriores; sin embargo, recientemente, ha hecho su aparición un nuevo tipo de tape con capacidades de amortiguación elástica denominado tape dinámico desarrollado por Ryan Kendrick, el cual, según sus propiedades parece tener ventajas de acción sobre sus predecesores (10). Por tal motivo, se hace necesario estudiar el efecto que el tape dinámico puede tener en la estática y función dinámica en mujeres con pie pronado.

\section{MATERIALES Y MÉTODOS}

Tipo de Estudio: investigación de tipo explicativa de carácter cuasi experimental.

Población: estudiantes de género femenino del Programa Ciencias del Deporte y la Recreación de la Universidad Tecnológica de Pereira.

Tipo de Muestreo: no probabilística intencionada de las estudiantes del Programa Ciencias del Deporte y la Recreación, que presenten pronación excesiva del pie con asignación aleatoria posterior a grupo experimental y grupo control.

Criterios de Inclusión: ser estudiantes activos de acuerdo a criterios de IPAQ corto, poseer características de pie pronado y pertenecer al Programa de Ciencias del Deporte y la Recreación de la Universidad Tecnológica de Pereira.

Criterios de Exclusión: antecedentes de alergia en piel que impidan el uso del tape dinámico y antecedentes quirúrgicos o patologías que imposibiliten el desarrollo de test funcionales. 


\section{Variables}

Dependientes: prueba de caída navicular e índice de postura del pie, las cuales determinan la estática de la postura del pie y la evaluación dinámica de la función del pie.

Independiente: técnica de aplicación del tape dinámico para corregir el ángulo longitudinal del pie.

Téenica e instrumentos para la recolección de los datos: se utilizarán pruebas dinámicas para miembro inferior y la medición del arco longitudinal medial. Se efectuará un análisis de diferencia de medias intra e intergrupos, previa comprobación de la normalidad de los datos para determinar los efectos del tape dinámico en la funcionalidad dinámica y en la estática del tobillo.

Resultados esperados: el desempeño de la función dinámica y estática del tobillo en mujeres con características de pie pronado mejora con el uso del tape dinámico.

Optimizar la funcionalidad biomecánica del tobillo y del pie disminuyendo la prevalencia de lesiones en miembros inferiores asociadas a la pronación excesiva del pie.

\section{IMPACTOS}

Personal: mejora del patrón de movimiento en tareas funcionales durante la realización de actividades básicas cotidianas, actividades de la vida diaria y actividades deportivas $\mathrm{y} / \mathrm{o}$ recreativas.

Social: contribución académica mediante la documentación de los mecanismos, pruebas y resultados obtenidos en el uso del tape dinámico, detallando secuencialmente el proceso para que pueda ser considerado por otros profesionales.

Optimización de los procesos de reaprendizaje motor ante la presencia de patrones alterados de movimiento acortando el tiempo de readquisición de patrón motor.

Potenciación de la terapia física convencional por la adición de medidas terapéuticas por la aplicación del tape dinámico.

Acortamiento del periodo de rehabilitación.

Económico: disminución de costos por consultas médicas, terapias físicas y procedimientos quirúrgicos de miembros inferiores asociados a las alteraciones 
1. Neal, B., Griffiths, I., Dowling, G., Murley, G., Munteanu, S., Franettovich, M., Collins, N., \& Barton, C., (2014). Foot posture as a risk factor for lower limb overuse injury: a systematic review and meta-analysis. Journal of Foot and Ankle Research, (7);55. https://doi.or/10.1186/s13047-014-0055-4

2. Newell, T., Simon, J., \& Docherty, C. L. (2015). Arch-taping techniques for altering navicular height and plantar pressures during activity. Journal of Athletic Training, 50(8), 825-832. https://doi.org/10.4085/1062-6050-50.5.05

3. Luque-Suarez, A., Gijon-Nogueron, G., Baron-Lopez, F. J., LabajosManzanares, M. T., Hush, J., \& Hancock, M. J. (2014). Effects of kinesiotaping on foot posture in participants with pronated foot: A quasi-randomised, doubleblind study. Physiotherapy (United Kingdom), 100(1), 36-40. https://doi. org/10.1016/j.physio.2013.04.005.

4. Lee, J., Yoon, J., \& Cynn, H. (2017). Foot exercise and taping in patients with patellofemoral pain and pronated foot. Journal of Bodywork and Movement Therapies, 21(1), 216-222. https://doi.org/10.1016/j.jbmt.2016.07.010

5. Chuter, V. H., \& Janse de Jonge, X. A. K. (2012). Proximal and distal contributions to lower extremity injury: A review of the literature. Gait and Posture, 36(1), 7-15. https://doi.org/10.1016/j.gaitpost.2012.02.001

6. Chuter,V. (2010). Relationships between foot type and dynamic rearfoot frontal plane motion. Journal of foot and ankle research. https://doi.org/10.1186/17571146-3-9

7. Aguilar, M. B., Abián-Vicén, J., Halstead, J., \& Gijon-Nogueron, G. (2016). Effectiveness of neuromuscular taping on pronated foot posture and walking plantar pressures in amateur runners. Journal of Science and Medicine in Sport, 19(4), 348-353. https://doi.org/10.1016/j.jsams.2015.04.004

8. Souza, T. R., Araújo, V. L., Silva, P. L., Carvalhais, V. O. C., Resende, R. A., \& Fonseca, S. T. (2016). External rotation elastic bands at the lower limb decrease rearfoot eversion during walking: a preliminary proof of concept. Braz J Phys Ther, 20(6), 571-579. https://doi.org/10.1590/bjpt-rbf.2014.0194

9. Simon, J., Garcia, W., Docherty, C., (2014) The Effect of Kinesio Tape on Force Sense in People With Functional Ankle Instability. Clinical Journal of Sport Medicine, 24(4), 289-294.https://doi.or/10.1097/JSM.0000000000000030

10. McNeill W, Pedersen C, Dynamic tape. Is it all about controlling load? J Bodyw Mov Ther. 2016;20 (1):179-88. https://doi.or/10.1016/j.jbmt.2015.12.009 